\title{
Childhood glaucoma registry in Germany: initial database, clinical care and research (pilot study)
}

\author{
Fidan A. Aghayeva ${ }^{1,2}$, Alexander K. Schuster ${ }^{1}$, Heidi Diel ${ }^{1}$, Panagiotis Chronopoulos ${ }^{1}$, Felix M. Wagner ${ }^{1}$,
} Franz Grehn ${ }^{1,3}$, Nina Pirlich ${ }^{4}$, Susann Schweiger ${ }^{5}$, Norbert Pfeiffer ${ }^{1}$ and Esther M. Hoffmann ${ }^{1 *}$ (D)

\begin{abstract}
Objective: The aim of this prospective pilot study is to establish an initial database to register patients diagnosed with different types of childhood glaucoma and the set-up of a national registry for childhood glaucoma (ReCG) in Germany. 28 children with different types of diagnosed childhood glaucoma, who were admitted and treated at the Childhood Glaucoma Center of the University Medical Center Mainz, Germany were included. Main outcome measures were the type of childhood glaucoma, mean intraocular pressure (IOP) and genetic data of the patients.

Results: The documents and questionnaires for each individual included: informed consent form of the parents, medical history form of the child, patient's gestational history questionnaire and general anesthesia examination form. Primary congenital and secondary childhood glaucoma were revealed in 11 (39\%) and 17 (61\%) patients, respectively. The mean IOP measured with Perkins tonometer in all patients under general anesthesia at the time of inclusion was $17.5 \pm 11.8 \mathrm{mmHg}$ in the right and $17 \pm 8.9 \mathrm{mmHg}$ in the left eyes. In 33\% of children with glaucoma mutations in the CYP1B1, FOXC1, LTBP2 and TEK genes were found. The development of specific questionnaires for childhood glaucoma provides detailed baseline data to establish a ReCG in Germany for the first time.
\end{abstract}

Keywords: Childhood glaucoma registry, Congenital glaucoma, Consanguinity, Genetic examination, Questionnaire

\section{Introduction}

Childhood glaucoma is a varied group of rare serious diseases. The incidence of this disorder differs regionally. Approximately one case occurs in 10,000-38,000 live births in Europe, North America, and Australia [1-6]. The highest prevalence can be found in slovakian gypsies (1:1250) [7], in Saudis (1:3030) [1,8] and in Southern India (1:3300) [9]. Childhood glaucoma is responsible for more than $18 \%$ of childhood blindness worldwide [3, $10-14]$.

*Correspondence: ehoffman@uni-mainz.de

${ }^{1}$ Department of Ophthalmology, University Medical Center

of the Johannes Gutenberg, University Mainz, Mainz, Germany

Full list of author information is available at the end of the article
Hence, childhood glaucoma is a rare disease in most regions, and etiology and pathogenesis of this disease are only partly known. The standard treatment of congenital glaucoma is the surgical opening of the angle structures to lower intraocular pressure (IOP) [15-17]. The available epidemiological, clinical and therapeutic data on childhood glaucoma are still insufficient [18].

To provide high quality clinical care for patients with childhood glaucoma in Germany, the Pediatric Glaucoma Center was inaugurated at the Department of Ophthalmology of the University Medical Center Mainz, Germany in June 2017. This Centre became soon a competence center taking care of childhood glaucoma patients not only from Germany, but also referred from all over Europe. The aim of this pilot study was to create an initial database of children with glaucoma referred 
and treated in our Center with maintenance of a national registry for childhood glaucoma (ReCG) in Germany that could be used by other hospitals worldwide. We report demographic, clinical and genetic data of first registered children with diagnosed childhood glaucoma.

\section{Main text \\ Materials and methods}

This pilot study included an initial dataset from 29 patients (58 eyes) with different types of childhood glaucoma or childhood glaucoma suspects, diagnosed and treated at the Children Glaucoma Center of the University Medical Center Mainz, Germany from December 2018 to December 2019. The study adhered to the tenets of the Declaration of Helsinki and was approved by the Ethics Committee of the Medical Board of RhinelandPalatinate, Germany.

A major aim of this pilot study was the development of forms and questionnaires to assess the feasibility and validity before building up a Germany-wide childhood glaucoma registry. These forms and questionnaires were developed and used for acquisition and storage of epidemiological (social data, gestational history, family history) and clinical data of patients diagnosed with childhood glaucoma. The patient information was stored in a database table-electronic form designed as a registry. All the demographic parameters were collected during outpatient examination; clinical parameters were obtained during ophthalmological outpatient examination or examination under general anesthesia.

In our analysis we used congenital glaucoma classification of the World Glaucoma Association [19], that categorizes primary congenital glaucoma (PCG) and the secondary childhood glaucoma (SCG) types, which are associated with other ocular and systemic findings [20, 21].

The health-related quality of life Questionnaires for children at age more than 3 years and adolescents up to 17 years of age (KINDL Questionnaires, Ravens-Sieberer \& Bullinger, 2000) were obtained from all included children, depending on their age (from 3 to 6 years; 7-13 years and 14-17 years). The KINDL Questionnaires contained basic questions on age, sex, number of siblings, school education and questions about health-related issues, connected with the glaucoma disease [22].

Indication for surgery was made on a clinical basis under anesthesia, considering the presence of two or more criteria of newly diagnosed children glaucoma or the progression of known disease, according to a novel classification system proposed by the new Childhood Glaucoma Research Network (CGRN) International Childhood Glaucoma Registry. The decision for surgery was immediately discussed with parents in each individual case and informed consent was obtained [21].

To better understand the role of inheritance we performed blood genetic testing of 25 (86.2\%) registered children and their parents to identify one or more of the known 24 genes, associated with congenital glaucoma (CNTNAP2, COL11A1, COL4A1, CYP1B1, FOXC1, FOXE3, LMX1B, LOXL1, LTBP2, MAF, MYOC, OPA1, OPA3, OPTN, PAX6, PCMTD1, PITX2, PITX3, PLEKHA7, ST18, TBK1, TEK, TMEM126A, WDR36). For this examination a minimum of $5 \mathrm{ml}$ Ethylenediamine tetraacetic acid (EDTA)-blood from the child under anesthesia ( $2 \mathrm{ml}$ if newborn or younger than 6 months) and a blood sample $(18 \mathrm{ml})$ from parents was taken [23]. The blood is stored anonymously for up to 10 years according to the German law for biomaterial storage in university medical centers. Besides, the genealogical tree was obtained for every case including special emphasis on consanguinity in the family (a blood relationship between the parents or grandparents).

\section{Statistical analysis}

Absolute and relative frequencies were computed for dichotomous data, continuous data are presented as mean \pm standard deviation. All statistical analysis was conducted with R (R Core Team 2020, R Foundation for Statistical Computing, Vienna, Austria).

\section{Results}

The created forms and questionnaires included:

1. Agreement form from the parents to participate in the pilot study with purpose of creating ReCG and storing and evaluating the study-related personal health data of their child in a pseudonymized way (i.e., coded without stating name, address, initials etc.,) in paper and electronic form on a secure server of the Mainz University Medical Center as well as a separate agreement form for children younger or older than 12 years. These forms were provided for the parents along with a detailed information letter and the oral explanation of the disease including treatment, registration process (the nature, significance, risks and scope of the register) and other logistic steps, such as possibility to withdraw the participation in the registry at any time, verbally or in writing, without giving any reasons, and without incurring any disadvantages for their child. It was mentioned, that collected data and biomaterial would be deleted after the end of the study, at the latest after 10 years, or at the time of withdrawal from the study.

2. Medical history form/sheet with medical and social history of the child, including patient registration 
number; birth date; sex; place of birth; place of residence; date and place, where diagnosis of glaucoma was first suspected; date of first examination by eye specialist; affected eye (right, left or both eyes); type of intended therapy (drops or surgery/laser), other diseases and use of medications (see Additional file 1: Fig. S1).

3. Patient's gestational history questionnaire (general information about pregnancy and details of delivery) including birth dates of both parents, parents' consanguinity, if present, total number of their biological children, history of childhood or juvenile glaucoma in their families (see Additional file 2: Fig. S2). This survey aimed at possible connections between risk factors in pregnancy and the incidence of childhood glaucoma. It could be completed within about $15 \mathrm{~min}$.

4. Form documenting the examination of the child under general anesthesia (GAEF), containing separate fields for: IOP measured with a hand-held applanation tonometer (Perkins, Haag-Streit, UK) and (Icare, Icare, USA); axial length measured by A-scan ultrasonography; central cornea thickness (AL-3000 Biometer/Pachymeter, Tomey Co, Nagoya, Japan and $\mathrm{iPac}^{\mathrm{TM}}$ Pachymeter Reichert Inc., USA); corneal diameter-horizontal and vertical; refraction and keratometry parameters (radius of corneal curvature) (Retinomax K-plus2, Nikon Inc., Japan); examination of anterior segment of the eye under the operating microscope (corneal haze, opacification, scarring, Haab's striae, iris, anterior chamber, and lens abnormalities); gonioscopy; fundus examination with pupil dilation and assessment of optic nerve disc and cupto-disc ratio (indirect ophthalmoscope, 20 Diopter lens); ultrasound B/scan image (Fig. 1).

In order to reduce interference of depth of anesthesia with IOP values $[24,25]$, IOP was always measured within the first minutes after initiation of anesthesia. Time point of IOP measurements, method of anesthesia, detailed description of anterior segment and gonioscopy findings, surgical status of the eye and signs of secondary glaucoma were thoroughly documented in the extended GAEF (see Additional file 3: Fig. S3). Examination under anesthesia was performed in 23 (79\%) patients. Fundus examination was difficult to perform in 29 (50\%) eyes, because of poor view to the posterior segment due to opacity of ocular media. In one patient with large cornea the glaucoma diagnosis was rejected during examination under anesthesia and a CHRDL1 gen, associated with megalocornea, was later identified during genetic blood examination. Demographic and clinical data of all patients with diagnosed childhood glaucoma are shown in Tables 1 and 2. Childhood glaucoma diagnosis was confirmed by considering all ocular, associated ocular, and systemic anomalies. PCG and SCG were revealed in $11(39 \%)$ and 17 (61\%) childhood patients, respectively. Bilateral glaucoma was diagnosed in 9 (82\%) and 10 (59\%) patients with PCG and SCG, respectively. The most common cause of SCG was Peters anomaly (41\%) (see Additional file 4: Table S1).

In $8(33 \%)$ children with childhood glaucoma one of the following congenital glaucoma associated genes (CYP1B1, FOXC1, LTBP2 and TEK) was confirmed by the genetic examination. The most frequently identified mutated gene was CYP1B1 located on chromosome 2p22-p21 (locus GLC3A) (Table 1, see Additional file 4: Table S1). In total, 24 (86\%) patients received IOP-lowering therapy and 17 (61\%) children underwent glaucoma surgery (Table 2). In 8 (29\%) childhood patients were performed multiple glaucoma surgeries.

One patient with SCG associated with Peters anomaly underwent combined XEN-Baerveldt drainage device implantation followed by revisions and lensectomy with synechiolysis after multiple glaucoma surgeries, including Ahmed valve implantation. We performed anterior chamber washout in one patient with hyphema after catheter-assisted $360^{\circ}$ trabeculotomy.

Due to inability of IOP control, in total, 4 (24\%) out of 17 patients underwent revisions or repeat glaucoma surgeries within 6 months after initial surgery in our Centre.

\section{Discussion}

Our pilot investigation describes the features of the first registry for childhood glaucoma in Germany. We developed special forms and questionnaires for data acquisition that can be used to ascertain the demographic and clinical characteristics of different types of childhood glaucoma.

There are different registries in almost all other areas of ophthalmology worldwide [26-31], including several glaucoma registries [32-35], but only few congenital glaucoma registries $[1,3,21]$.

Lopes et al. designed an online form at the Department of Ophthalmology and Visual Sciences of the Federal University of São Paulo to create a registry of patients with congenital glaucoma. The pilot study included 72 children with childhood glaucoma, $61.5 \%$ with PCG, and $38.5 \%$ with secondary congenital glaucoma, respectively. Most patients with secondary congenital glaucoma were secondary to congenital cataract (61\%) [3]. In contrast, most of our SCG patients had Peters anomaly.

Thau et al. presented a review of a new classification system for childhood glaucoma, its use to date, and its application for international research. This classification has become the first International Consensus 
Classification in 2013 and is used by the new Robison D. Harley, MD CGRN International Pediatric Glaucoma Registry. This registry attempts to overcome the inherent challenges of studies on rare disorders due to limited study size and acts as a centralized database for clinical data provided by sites across the globe to allow largescale studies on childhood glaucoma [21].

Alanazi et al. determined the demographic and clinical distribution of primary and secondary congenital glaucoma in 180 patients with congenital glaucoma in the
EXAMINATION UNDER GENERAL ANESTHESIA:

Clinic: Department of Ophthalmology of the University Medical Center Mainz
Date

Doctor:
ID:

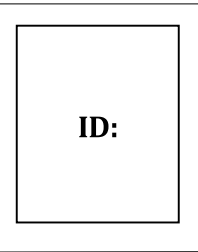

a) Intraocular pressure (mmHg): after induction of anesthesia
iCare
OD:
OS:
Perkins OD:
OS:

b) Axial length ( $\mathrm{mm}$ ): Tomey

OD:

OS: c) Pachymetry $(\mu \mathrm{m})$ : Tomey

please attach

d) Corneal diameter $(\mathrm{mm})$ :

OD: horizontal: vertical:

OS: horizontal: vertical:

Fig. 1 General anesthesia examination form (GAEF) 
Middle East by using data from a registry at King Khaled Eye Specialist Hospital, collected between 2001 and 2003 (29 months). This registry excluded cases with acquired childhood glaucoma. According to their results, $30 \%$ of all patients had a positive family history and consanguinity was recognized among $58.9 \%$ patients [1].

Different from Arabic and Pakistani populations in which consanguineous marriages are the major

please attach

please attach

ID:

e) Refractometry [REF] \& Keratometry [KER] : Retinomax

f) Anterior segment:

Description:

Fig. 1 continued 


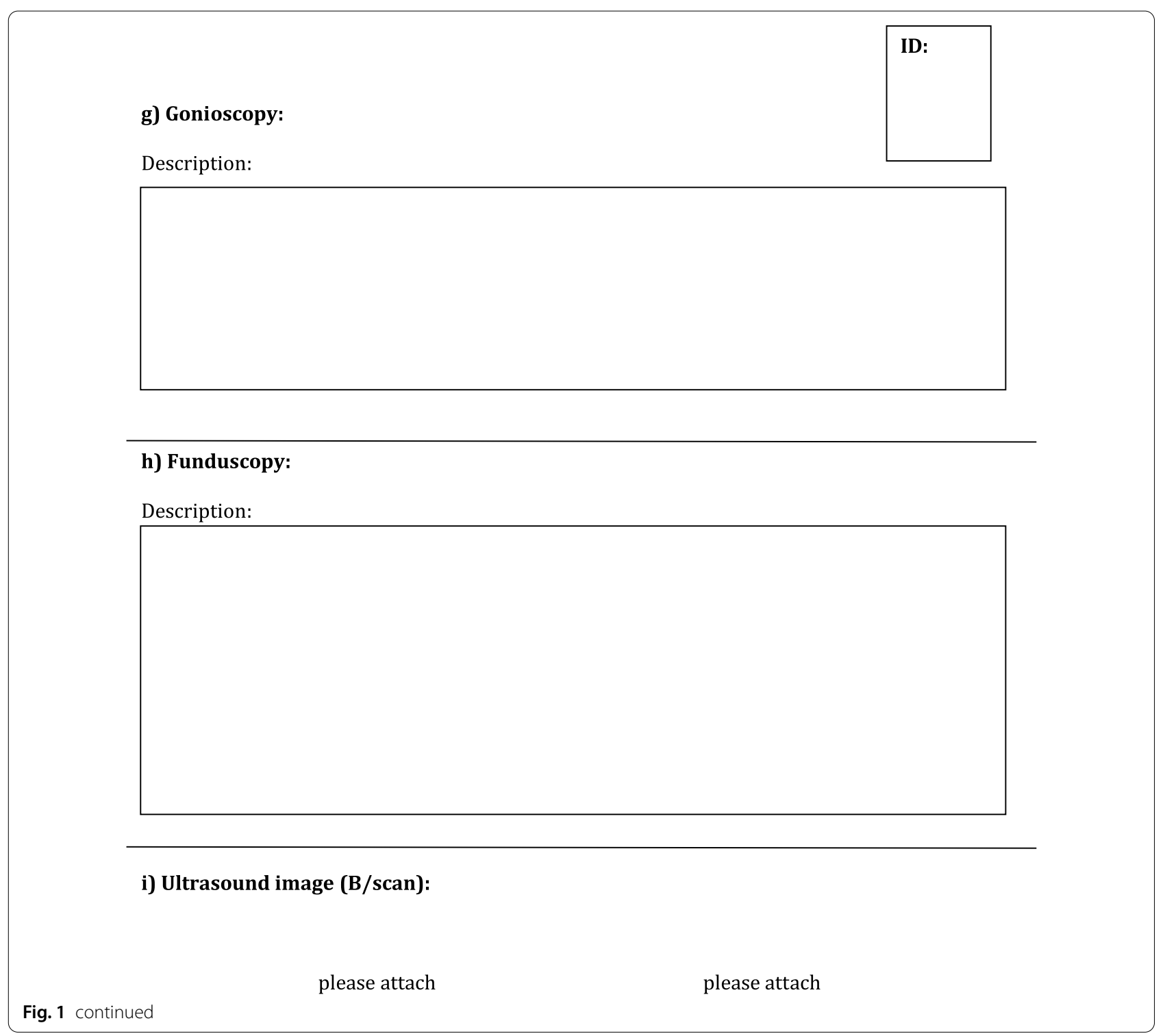

contributing factors to the high incidence of recessively inherited PCG $[1,5,14,36]$, we found a much lower frequency of consanguinity in our study ( $21 \%$ of positive family history and $14 \%$ of presence of consanguineous marriages). These percentages are comparable to those, reported in the U.S. and Australia [2, 4]. In one third of included childhood glaucoma patients genetic testing identified one of the genes that are associated with congenital glaucoma, confirming the importance of genotyping this disease $[7,36]$.

Bilateral disease was present in our study in $82 \%$ patients with PCG and was higher than the incidence of bilateral PCG (58\%), reported by Bayoumi [37]. We observed unilateral disease more frequent in patients with SCG than in PCG, as previously reported $[1,20,38]$.

\section{Conclusions}

In summary, this pilot study at the Children Glaucoma Center of the University Medical Center Mainz provided for the first time in Germany detailed baseline data and is aimed at a national registry on different types of childhood glaucoma.

The planned registry will allow to perform various types of registry-based analyses as well as a better understanding of the clinical course of the disease and identification of risk factors. Registered data can provide valuable 
Table 1 Descriptive statistics of demographic and genetic data of all patients with diagnosed childhood glaucoma

\begin{tabular}{|c|c|}
\hline Number of patients, PCG/SCG & $28 ; 11 / 17$ \\
\hline \multicolumn{2}{|l|}{ Age at presentation } \\
\hline Mean \pm SD (range), months & $33.8 \pm 36.5(1-125)$ \\
\hline Mean \pm SD (range), years & $2.8 \pm 3(0.07-10.41)$ \\
\hline \multicolumn{2}{|l|}{ Age at time of suspected glaucoma diagnosis, months } \\
\hline Mean \pm SD (range) & $12.3 \pm 24.9(0-119.3)$ \\
\hline \multicolumn{2}{|l|}{ Sex, n (\%) } \\
\hline Female/Male & $18(64 \%) / 10(36 \%)$ \\
\hline \multicolumn{2}{|l|}{ Country of origin, mother/father, $\mathrm{n}(\%)$} \\
\hline German & $14(50 \%) / 14(50 \%)$ \\
\hline East European countries & $5(18 \%) / 6(21 \%)$ \\
\hline Asia & $7(25 \%) / 7(25 \%)$ \\
\hline Africa & $2(7 \%) / 1(4 \%)$ \\
\hline \multicolumn{2}{|l|}{ Country of birth, $\mathrm{n}(\%)$} \\
\hline German & $21(75 \%)$ \\
\hline East European countries & $4(14 \%)$ \\
\hline Asia & $3(11 \%)$ \\
\hline \multicolumn{2}{|l|}{ Laterality, n (\%) } \\
\hline Unilateral/Bilateral & $9(32 \%) / 19(68 \%)$ \\
\hline Unilateral/Bilateral, PCG & $2(18 \%) / 9(82 \%)$ \\
\hline Unilateral/Bilateral, SCG & $7(41 \%) / 10(59 \%)$ \\
\hline Mean number of performed eye surgeries & $2 \pm 3.7$ \\
\hline Mean number of eye surgeries, performed at glaucoma department Mainz & $2 \pm 3.2$ \\
\hline Positive family history, $\mathrm{n}(\%)$ & $6(21 \%)$ \\
\hline Consanguinity, n (\%) & $4(14 \%)$ \\
\hline \multicolumn{2}{|l|}{ Risk factors during pregnancy, $\mathrm{n}(\%)$} \\
\hline Smoking & $2(7 \%)$ \\
\hline Alcohol drinking & $3(11 \%)$ \\
\hline Genetic examination & $24(86 \%)$ \\
\hline Revealed genes' mutations with their type and location, n (\%) & $12(50 \%)$ out of 24 \\
\hline CYP1B1 (2 compound heterozygous; 3 homozygous; 2p22.2) & $5(21 \%)$ \\
\hline SOX11 (heterozygous; 2p25.2) & $1(4 \%)$ \\
\hline TEK (heterozygous; 9p21.2) & $1(4 \%)$ \\
\hline CRYBB3 (heterozygous; 22q11.23) & $1(4 \%)$ \\
\hline FYCO1 (homozygous; 3p21.31) & $1(4 \%)$ \\
\hline FOXC1 (heterozygous; 6p25.3) & $1(4 \%)$ \\
\hline GJA8 (heterozygous; 1q21.2) & $1(4 \%)$ \\
\hline LTBP2 (compound heterozygous; 14q24.3) & $1(4 \%)$ \\
\hline
\end{tabular}

SD, standard deviation; PCG, primary congenital glaucoma; SCG, secondary childhood glaucoma

information on epidemiology, clinical phenotypes and genetics in childhood glaucoma blindness in Germany.

\section{Limitations}

The limitation of this registry was the collection of medical history only by an interview with the child's parents and not by review of their medical documentation which was not available in most cases. Hence, no complete verification of the family and gestational history is possible in the questionnaires. Due to the lack of a documented description of previous surgical techniques, in most cases it was also impossible to confirm the use of certain surgical agents and conduct a detailed descriptive analysis of interventions performed. We did not investigate differences in age, sex, and ethnical distribution in our study cohort, since it is a pilot study with a limited number of patients in both the PCG and SCG group. 
Table 2 Descriptive statistics of clinical data of patients $(n=28)$ with diagnosed childhood glaucoma

\begin{tabular}{|c|c|c|}
\hline Data/eye & Right, $n=23$ & Left, $n=24$ \\
\hline \multicolumn{3}{|l|}{ Type of childhood glaucoma } \\
\hline PCG & 9 & 11 \\
\hline SCG & 14 & 13 \\
\hline Presence of IOP-lowering therapy & $19(83 \%)$ & $21(88 \%)$ \\
\hline Topical monotherapy & $6(26 \%)$ & $5(21 \%)$ \\
\hline Topical combined therapy & $12(52 \%)$ & $15(62 \%)$ \\
\hline 2 groups of hypotensive drops & $9(39 \%)$ & $8(33 \%)$ \\
\hline$\geq 3$ groups of hypotensive drops & $3(13 \%)$ & $7(29 \%)$ \\
\hline Acetacolamide_-in one patient & $1(4 \%)$ & $1(4 \%)$ \\
\hline \multicolumn{3}{|l|}{ Surgical status of eye } \\
\hline Untreated & $11(48 \%)$ & $9(38 \%)$ \\
\hline Previous TO & $3(13 \%)$ & $3(13 \%)$ \\
\hline Previous TE & $1(4 \%)$ & $2(8 \%)$ \\
\hline Previous Coco or Cyclocryocoagulation & - & $1(4 \%)$ \\
\hline Previous multiple glaucoma surgeries & $7(30 \%)$ & $8(33 \%)$ \\
\hline Previous drainage device (Ahmed valve) & $1(4 \%)$ & $1(4 \%)$ \\
\hline \multicolumn{3}{|l|}{ Mean IOP (SD, range), mmHg } \\
\hline Perkins & $17.5 \pm 11.8(5-50)$ & $17 \pm 8.9(5-43)$ \\
\hline iCare & $23.3 \pm 18(7-68.5)$ & $20.6 \pm 9.4(8.2-38.7)$ \\
\hline Mean axial length (SD), mm & $22.3 \pm 3.5$ & $23.5 \pm 3.8$ \\
\hline \multicolumn{3}{|l|}{ Mean corneal diameter (SD), mm } \\
\hline Horizontal & $12.4 \pm 2.7$ & $11.9 \pm 2.5$ \\
\hline Vertical & $11.3 \pm 3.1$ & $10.9 \pm 3.2$ \\
\hline Mean CCT (SD), $\mu \mathrm{m}$ & $623 \pm 110.3$ & $632 \pm 123.7$ \\
\hline \multicolumn{3}{|l|}{ Refraction, diopters } \\
\hline Mean sphere (SD) & $1.43 \pm 9.8$ & $-0.23 \pm 8.6$ \\
\hline Mean cylinder (SD) & $-2.42 \pm 2.2$ & $-3.73 \pm 2.6$ \\
\hline \multicolumn{3}{|l|}{ Keratometry, mm } \\
\hline Mean K1 (SD) & $7.8 \pm 0.8$ & $8.4 \pm 0.8$ \\
\hline Mean K2 (SD) & $7.3 \pm 0.7$ & $7.9 \pm 0.8$ \\
\hline
\end{tabular}

PCG, primary congenital glaucoma; SCG, secondary childhood glaucoma; TO, trabeculotomy; TE, trabeculectomy; Coco, cyclophotocoagulation; SD, standard deviation; IOP, intraocular pressure; CCT, central corneal thickness

\section{Abbreviations}

ReCG: National registry for childhood glaucoma; IOP: Intraocular pressure; GAEF: General anesthesia examination form; PCG: Primary congenital glaucoma; SCG: Secondary childhood glaucoma; CGRN: Childhood Glaucoma Research Network; EDTA: Ethylenediamine tetraacetic acid.

\section{Supplementary Information}

The online version contains supplementary material available at https://doi. org/10.1186/s13104-022-05921-8.

Additional file 1: Figure S1. Medical history form with medical and social history of the child.

Additional file 2: Figure S2. Patient's gestational history questionnaire (general information about pregnancy and details of delivery).

Additional file 3: Figure S3. Extended general anesthesia examination form (extended GAEF). PHPV = Persistent hyperplastic primary vitreous; $\mathrm{ROP}=$ Retinopathy of prematurity; FEVR = Familial exudative vitreoretinopathy.
Additional file 4: Table S1. Causes and genetic data of secondary childhood glaucoma in Germany.

\section{Acknowledgements}

Not applicable.

\section{Authors' contributions}

FA: investigation, visualization, formal analysis; writing —original draft preparation. AS: methodology; validation; formal analysis, writing — reviewing and editing. HD: data curation, investigation, visualization, writing — reviewing and editing. PC: methodology; writing-reviewing and editing. FW: writing - reviewing and editing. FG: conceptualization; methodology; resources; writing — reviewing and editing. NP: conceptualization, investigation, writing - reviewing and editing. SS: conceptualization, writing-reviewing and editing. NP: project administration, resources, writing — reviewing and editing. EH: conceptualization; methodology; resources; writing-reviewing and editing; supervision. All authors read and approved the final manuscript. 


\section{Funding}

Open Access funding enabled and organized by Projekt DEAL. This research did not receive any specific support from funding agencies in the public, commercial, or not-for-profit sectors.

\section{Availability of data and materials}

The authors confirm that the data supporting the findings of this study are available within the article and its Additional files. This manuscript contains part of the doctoral thesis of Dr. Heidi Diel named "Results of a pilot study at the Department of Ophthalmology of the University Medical Center Mainz to establish a nationwide registry for childhood glaucoma in Germany" at the University Medical Center of the Johannes Gutenberg-University Mainz. The registry datasets generated and analyzed during the current study are available from the corresponding author on reasonable request.

\section{Declarations}

\section{Ethics approval and consent to participate}

This database study involving human participants adhered to the tenets of the Declaration of Helsinki and was approved by the Ethics Committee of the Medical Board of Rhineland-Palatinate, Germany. Written informed consent to participate in this pilot study was obtained from parents for all participants under 16 years old.

\section{Consent for publication}

Not applicable.

\section{Competing interests}

The authors declare that they have no competing interests that are relevant to the content of this article.

\section{Author details}

${ }^{1}$ Department of Ophthalmology, University Medical Center of the Johannes Gutenberg, University Mainz, Mainz, Germany. ${ }^{2}$ National Centre of Ophthalmology Named After Academician Zarifa Aliyeva, Baku, Azerbaijan. ${ }^{3}$ Department of Ophthalmology, University Medical Center Würzburg, Würzburg, Germany. ${ }^{4}$ Department of Anaesthesiology, University Medical Center of the Johannes Gutenberg, University Mainz, Langenbeckstraße 1, 55131 Mainz, Germany. ${ }^{5}$ Institute of Human Genetics, University Medical Centre of the Johannes Gutenberg, University Mainz, Mainz, Germany.

Received: 30 October 2021 Accepted: 25 January 2022

Published online: 10 February 2022

\section{References}

1. Alanazi FF, Song JC, Mousa A, et al. Primary and secondary congenital glaucoma: baseline features from a registry at King Khaled Eye Specialist Hospital, Riyadh, Saudi Arabia. Am J Ophthalmol. 2013;155(5):882-9.

2. Aponte EP, Diehl N, Mohney BG. Incidence and clinical characteristics of childhood glaucoma: a population-based study. Arch Ophthalmol. 2010;128(4):478-82.

3. Lopes NLV, Gracitelli CPB, Moura CR. Creation of a childhood glaucoma registry database. Arq Bras Oftalmol. 2018;81(4):271-5.

4. MacKinnon JR, Giubilato A, Elder JE, Craig JE, Mackey DA. Primary infantile glaucoma in an Australian population. Clin Exp Ophthalmol. 2004;32(1):14-8.

5. Papadopoulos M, Cable N, Rahi J, Khaw PT. The British infantile and childhood glaucoma (BIG) eye study. Invest Ophthalmol Vis Sci. 2007:48(9):4100-6.

6. Taylor RH, Ainsworth JR, Evans AR, Levin AV. The epidemiology of childhood glaucoma: the Toronto experience. J AAPOS. 1999;3(5):308-15.

7. Gencik A, Gencikova A, Ferak V. Population genetical aspects of primary congenital glaucoma. I. Incidence, prevalence, gene frequency, and age of onset. Hum Genet. 1982;61(3):193-7.

8. Jaffar M. Care of the infantile glaucoma patient. In: Reinecke R, editor. Ophthalmology annual. New York: Raven Press; 1988. p. 15-37.
9. Dandona L, Williams JD, Williams BC, Rao GN. Population based assessment of childhood blindness in southern India. Arch Ophthalmol. 1998;116(4):545-6.

10. Chong C, McGhee CNJ, Dai SH. Causes of childhood low vision and blindness in New Zealand. Clin Exp Ophthalmol. 2019;47(2):165-70.

11. Gilbert CE, Canovas R, Hagan M, Rao S, Foster A. Causes of childhood blindness: results from west Africa, south India and Chile. Eye. 1993;7:184-8.

12. Kong L, Fry M, Al-Samarraie M, Gilbert C, Steinkuller PG. An update on progress and the changing epidemiology of causes of childhood blindness worldwide. J AAPOS. 2012;16(6):501-7.

13. Mezer E, Chetrit A, Kalter-Leibovici O, Kinori M, Ben-Zion I, WygnanskiJaffe T. Trends in the incidence and causes of severe visual impairment and blindness in children from Israel. J AAPOS. 2015;19(3):260-5.e1.

14. Tabbara KF, Badr IA. Changing pattern of childhood blindness in Saudi Arabia. Br J Ophthalmol. 1985;69(4):312-5.

15. Grehn F. Congenital glaucoma surgery: a neglected field in ophthalmology? Br J Ophthalmol. 2008;92:1-2.

16. Hoffmann EM. $360^{\circ}$ trabeculotomy for childhood glaucoma. Ophthalmologe. 2020;117(3):210-4.

17. Toshev AP, Much MM, Klink T, Pfeiffer N, Hoffmann EM, Grehn F. Catheter-assisted 360-degree trabeculotomy for Congenital glaucoma. J Glaucoma. 2018;27(7):572-7.

18. Ghate $D$, Wang $X$. Surgical interventions for primary congenital glaucoma. Cochrane Database Syst Rev. 2015;1: CD008213. https://doi.org/ 10.1002/14651858.CD008213.pub2.

19. Weinreb RN, Grajewski AL, Papadopoulos M, Grigg J, Freedman S, editors. Childhood glaucoma. WGA consensus series 9. Amsterdam: Kugler Publ; 2013.

20. Hoskins H Jr, Shaffer RN, Hetherington J. Anatomical classification of the developmental glaucomas. Arch Ophthalmol. 1984;102:1331-6.

21. Thau A, Lloyd M, Freedman S, Beck A, Grajewski A, Levin AV. New classification system for childhood glaucoma: implications for clinical care and a research registry. Curr Opin Ophthalmol. 2018;29(5):385-94.

22. Ravens-Sieberer U, Bullinger M. Assessing health related quality of life in chronically ill children with the German KINDL: first psychometric and content analytical results. Qual Life Res. 1998;7:399-407.

23. Banfi G, Salvagno GL, Lippi G. The role of ethylenediamine tetraacetic acid (EDTA) as in vitro anticoagulant for diagnostic purposes. Clin Chem Lab Med. 2007:45(5):565-76.

24. Obsa MS, Kanche ZZ, Olana Fite R, et al. Effect of laryngeal mask airway insertion on intraocular pressure response: systematic review and meta-analysis. Anesthesiol Res Pract. 2020;2020:7858434.

25. Termühlen J, Gottschalk A, Eter N, et al. Does general anesthesia have a clinical impact on intraocular pressure in children? Paediatr Anaesth. 2016;26(9):936-41.

26. Eid OM, Abdel Hady S, El-Kotoury A, Said KA, Rafat K, El-Bassyouni HT. Registry of ocular anomalies among patients with genetic disorders attending the clinical genetics department at the National Research Center in Egypt. Ophthalmic Genet. 2017;38(5):418-21.

27. Holmström G, Hellström A, Jakobsson P, Lundgren $P$, Tornqvist $K$, Wallin A. Five years of treatment for retinopathy of prematurity in Sweden: results from SWEDROP, a national quality register. $\mathrm{Br} J$ Ophthalmol. 2016;100(12):1656-61.

28. Hoque DM, Kumari V, Ruseckaite R, Romero L, Evans SM. Impact of clinical registries on quality of patient care and health outcomes: protocol for a systematic review. BMJ Open. 2016;6(4):e010654.

29. Lundström M, Stenevi U, Thorburn W. The Swedish National Cataract Register: a 9-year review. Acta Ophthalmol Scand. 2002;80(3):248-57.

30. Roth M, Holtmann C, Böhringer D, et al. Status and perspectives of registry-based studies in German ophthalmology. Ophthalmologe. 2020;117(1):36-43.

31. Tan JCK, Ferdi AC, Gillies MC, Watson SL. Clinical registries in ophthalmology. Ophthalmology. 2019;126(5):655-62.

32. Hohberger B, Monczak E, Mardin CY. 26 years of the erlangen glaucoma registry: demographic and perimetric characteristics of patients through the ages. Klin Monbl Augenheilkd. 2019;236(5):691-8.

33. Iester M, Telani S, Brusini P, et al. Italian Glaucoma Register. Central corneal thickness and glaucoma treatment: an Italian multicenter cross-sectional study. J Ocul Pharmacol Ther. 2013;29(5):469-73. 
34. Souzeau E, Goldberg I, Healey PR, et al. Australian and New Zealand registry of advanced glaucoma: methodology and recruitment. Clin Exp Ophthalmol. 2012;40(6):569-75.

35. Wolfram C, Pfeiffer N. Glaucoma diseases in Rhineland-Palatine 2010. Epidemiology and utilization of healthcare. Ophthalmologe. 2012;109(3):271-6.

36. Bejjani BA, Lewis RA, Tomey KF, et al. Mutations in CYP1B1, the gene for cytochrome P4501B1, are the predominant cause of primary congenital glaucoma in Saudi Arabia. Am J Hum Genet. 1998;62(2):325-33.

37. Bayoumi NH. Fellow eye in unilateral primary congenital glaucoma. J Curr Glaucoma Pract. 2017;11(1):28-30.

38. Khitri MR, Mills MD, Ying GS, Davidson SL, Quinn GE. Visual acuity outcomes in childhood glaucomas. J AAPOS. 2012;16(4):376-81.

\section{Publisher's Note}

Springer Nature remains neutral with regard to jurisdictional claims in published maps and institutional affiliations.

- fast, convenient online submission

- thorough peer review by experienced researchers in your field

- rapid publication on acceptance

- support for research data, including large and complex data types

- gold Open Access which fosters wider collaboration and increased citations

- maximum visibility for your research: over 100M website views per year

At BMC, research is always in progress.

Learn more biomedcentral.com/submissions 